\title{
DESPLAZAMIENTOS RELATIVOS LONGITUDINALES EN PILAS DE PUENTES
}

\author{
José Luis Estrada Ruiz ${ }^{(1)}$ y Eduardo Reinoso Angulo ${ }^{(1)}$
}

\section{RESUMEN}

Se presenta un estudio para la determinación de la longitud necesaria de apoyo de elementos de la superestructura a partir de la respuesta calculada de los desplazamientos relativos que se presentan entre las pilas de un puente estructurado a base de claros simplemente apoyados. El estudio se realiza considerando a los puentes desplantados en distintos tipos de suelo. La presencia de tales desplazamientos relativos originada por la diferencia en las respuestas de cada pila sometida a la acción de un sismo, puede llegar a producir golpeteo en los elementos de la superestructura y eventualmente el colapso de la misma por la pérdida de apoyo. Para la obtención de tales desplazamientos, se toman en cuenta varios parámetros como son la longitud del claro entre pilas y el periodo de vibrar de las mismas; la propuesta que se hace sobre la longitud mínima de apoyo está en función de tales parámetros.

\begin{abstract}
A study of the relative displacements between bridge piles and the required seating length of the elements of the bridge superstructure is presented. We first obtain the relative displacements between the piles due to recorded strong ground motion at different sites with different soil profiles for the Michoacán 1985 earthquake. The presence of such relative displacements originated by the different behavior of each pile and to different wave arrivals may lead to pounding between the bridge elements and eventually to the loss of support and collapse of the superstructure. To obtain the minimum support length for such bridge superstructures, we propose a parametric expression that considers, for each site, the span length and the period of vibration of the piles.
\end{abstract}

\section{INTRODUCCIÓN}

A partir de los sismos ocurridos en los últimos treinta años se ha observado que algunas estructuras especiales, como los puentes, han sufrido daños importantes (Priestley et al., 1996). Fue a partir del sismo de San Fernando en 1971 donde se presentaron estos daños, por lo que se tomaron medidas corrigiendo las normas de diseño vigentes en esa época. Al presentarse los sismos de Loma Prieta (1989) y Northridge (1994), ambos en California, ocurrieron nuevos daños, lo que condujo a una nueva revisión de las normas de diseño. En 1995 en Kobe, Japón,

Artículo recibido el 4 de noviembre de 2004 y aprobado para su publicación el 11 de febrero de 2005. Se aceptarán comentarios y/o discusiones hasta cinco meses después de su publicación.

(1) Instituto de Ingeniería, UNAM, Ciudad Universitaria, 04510 México, D.F., jluiser1@yahoo.com.mx, ere@pumas.iingen.unam.mx 
ocurrió otro sismo que causó gran daño en la ciudad. Dentro de los daños ocurridos en puentes y vialidades elevadas, se tienen los debidos al colapso por pérdida de apoyo de la superestructura como se muestra en la figura 1 para varios sismos recientes por causa de los grandes desplazamientos relativos entre las pilas en dirección longitudinal.
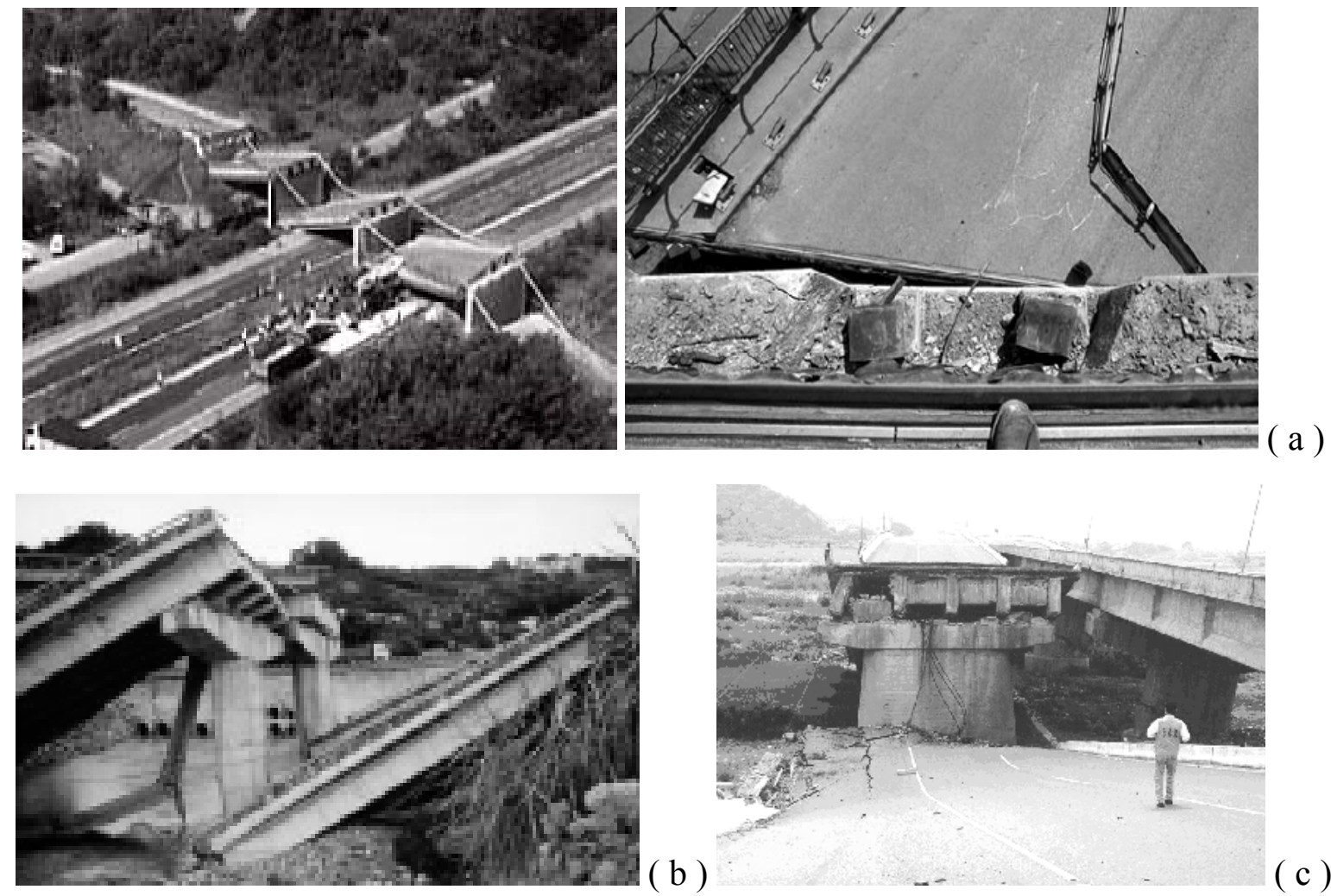

(c)
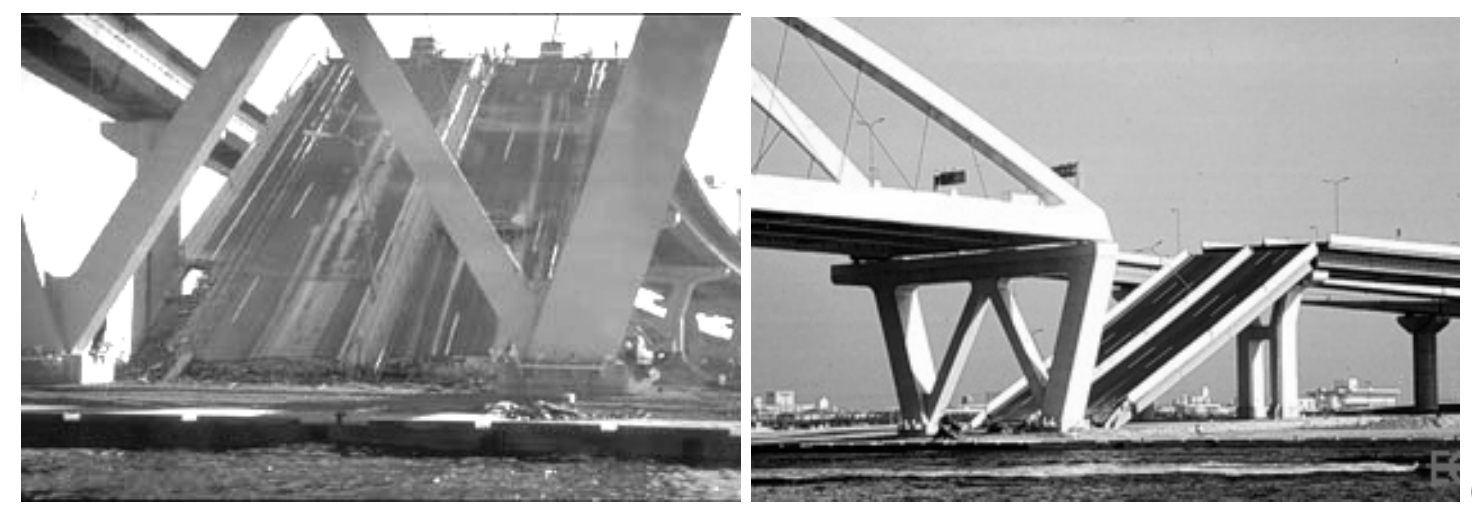

( d )

Figura 1. Colapsos en puentes durante sismos recientes de elementos simplemente apoyados: (a) Sismo de Kocaeli, Turquía, agosto 17, 1999; se aprecia en la foto de la derecha el reducido tamaño de los neoprenos $(10-12 \mathrm{~cm})$ y de la longitud de apoyo $(12-15 \mathrm{~cm})$. (b) Sismo de Chi-Chi, Taiwán, septiembre 21, 1999; puente Shi-Wei. (c) Sismo de ChiChi, Taiwán, septiembre 21, 1999, puente Wu-Shi; obsérvese el daño por golpeteo en la porción de la superestructura que no colapsó. (d) Sismo de Kobe, Japón, enero 17, 1995, puente Nishinomiya. 
Los sismos ocurridos en el año de 1999 en Turquía y Taiwán, y en años posteriores en distintos países del mundo, han provocado daños en este tipo de estructuras, que ponen de manifiesto la necesidad de estudiar de manera detallada su comportamiento para obtener diseños que conduzcan a un mejor desempeño, ya que la pérdida de operación de una estructura de este tipo entorpecen de forma importante las acciones a llevar a cabo después de la ocurrencia de un evento de este tipo.

Existen algunos trabajos que abordan el estudio de los desplazamientos relativos entre pilas de puentes con estructuración a base de claros simplemente apoyados, tales como DesRoches y otros (2002) y Malhotra (1998), que se enfocan a la evaluación del daño causado por el golpeteo entre elementos de la superestructura. De estos trabajos hemos tomado la forma de modelar el comportamiento de este tipo de estructuras al producirse golpeteo durante sismos.

Hao (1998) realiza un estudio paramétrico para evaluar la longitud necesaria de apoyo en puentes a partir de la diferencia de respuestas entre las pilas mediante análisis en el dominio de la frecuencia. Considera excitaciones en la base de cada soporte no uniformes, las cuales modela con una función espectral de potencia y una función de coherencia empírica. Dicho estudio muestra resultados obtenidos del análisis de un modelo de un puente de dos claros en función de ciertas propiedades del puente tales como los periodos asociados a cada claro del puente, la longitud de los mismos y las propiedades dinámicas del suelo, sometidos a una aceleración pico en el suelo, mostrando las tendencias de las respuestas del puente ante estos parámetros.

En este contexto, existen algunas expresiones de uso común propuestas en diferentes códigos de diseño tales como AASTHO y CALTRANS, las cuales son aplicables a la mayoría de los casos. No obstante por las características tan especiales que presenta el suelo de la ciudad de México es importante realizar estudios propios sobre el efecto de la acción sísmica en los puentes en donde se mejoren las expresiones contenidas en los citados manuales.

Del trabajo que aquí se presenta se encontró que el factor principal que determina la longitud de apoyo necesaria es la relación de periodos de vibrar de las pilas adyacentes. Sin embargo, las variaciones en el movimiento del suelo en cada soporte cuando los periodos de vibrar son muy parecidos son un factor muy importante para determinar la longitud de apoyo. Dicha longitud se incrementa cuando el periodo de vibrar del puente coincide con el periodo dominante del suelo.

\section{FACTORES QUE INFLUYEN EN LOS DESPLAZAMIENTOS RELATIVOS}

Un puente sometido a las acciones provocadas por un sismo puede experimentar diferencias de desplazamiento horizontal en la subestructura tanto en dirección longitudinal como en transversal debido a varios factores. Según la configuración de la estructuración se pueden tener dos casos, uno donde la estructura sea continua, es decir, que la superestructura esté unida monolíticamente con cada elemento de la subestructura, y el segundo caso cuando cada elemento de la subestructura es independiente de los demás. En este segundo caso, en el que se enfoca este estudio, los elementos de la superestructura se encuentran simplemente apoyados sobre los 
elementos que componen la subestructura, por lo que tienen cierta libertad de desplazamiento horizontal.

La respuesta lineal de una estructura sometida a una acción dinámica depende de propiedades como el periodo de vibrar y el amortiguamiento. Al comparar la respuesta de dos estructuras, las diferencias entre las respuestas dependerá de esas propiedades; además, existen otros factores que dependen de la geometría del puente y las propiedades del suelo donde se desplanta la estructura que influyen de manera importante en los desplazamientos relativos de la misma.

\section{Aspectos estructurales}

Al presentarse diferencias en la geometría de las pilas, estas no tendrán la misma rigidez y por consecuencia tendrán diferente periodo de vibrar, por lo que se presentarán respuestas también diferentes entre si. Tal es el caso de los puentes irregulares mostrados en la figura 2 que presentan variaciones en la altura de las pilas y en la longitud de los claros. Otro factor del cual depende el periodo de vibrar es la masa de la estructura, que en el caso de puentes varía de una pila a otra en función de longitud de separación entre estas.
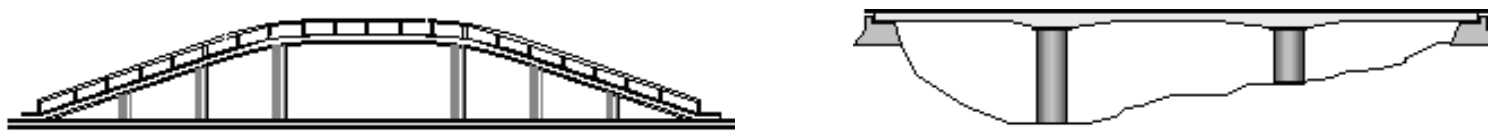

Figura 2. Puentes con cambios de rigidez en las pilas de la subestructura.

\section{Efecto del paso de las ondas}

Las ondas sísmicas arriban a cada pila en tiempos diferentes, lo cual depende de la geometría del puente y del ángulo de arribo de las ondas. Ese ángulo, con respecto al eje longitudinal del puente, genera una diferencia en el tiempo de llegada de la onda entre las pilas que se puede expresar mediante la siguiente ecuación obtenida a partir de lo expuesto en la figura 3 ,

$$
\Delta t=\frac{\text { distancia }}{\text { velocidad }}=\frac{L \cdot \cos \theta}{V_{w}}
$$

donde $L$ es la longitud entre apoyos, $V_{w}$ es la velocidad de la onda sísmica en el suelo, $\theta$ es el ángulo de llegada de la onda sísmica con respecto al eje longitudinal del puente y $\Delta t$ es la diferencia de tiempo de llegada de la onda sísmica entre pilas. En este aspecto la velocidad de propagación de las ondas sísmicas en el suelo tiene una importancia relevante particularmente cuando dicha velocidad es relativamente baja. La velocidad de propagación de las ondas de cuerpo depende de la densidad y propiedades elásticas del suelo. Con respecto a las ondas 
superficiales, considerando un semiespacio elástico homogéneo, la velocidad de las ondas de Rayleigh y de Love es cercana a la de las ondas de corte y es función de la frecuencia; Roullé (2004) muestra la variación de estas velocidades con respecto a la frecuencia en sitios de suelo blando del valle de México, comprobando que dichas velocidades son cercanas a la velocidad de las ondas de corte y permanecen relativamente constantes en el intervalo de periodos de interés $(0.3-1.3 \mathrm{seg})$. Previamente, Reinoso (2002) mostró que las ondas de cuerpo, tanto las ondas $\mathrm{P}$ como las $\mathrm{S}$, y las superficiales (Rayleigh) producen efectos similares en la superficie del suelo.

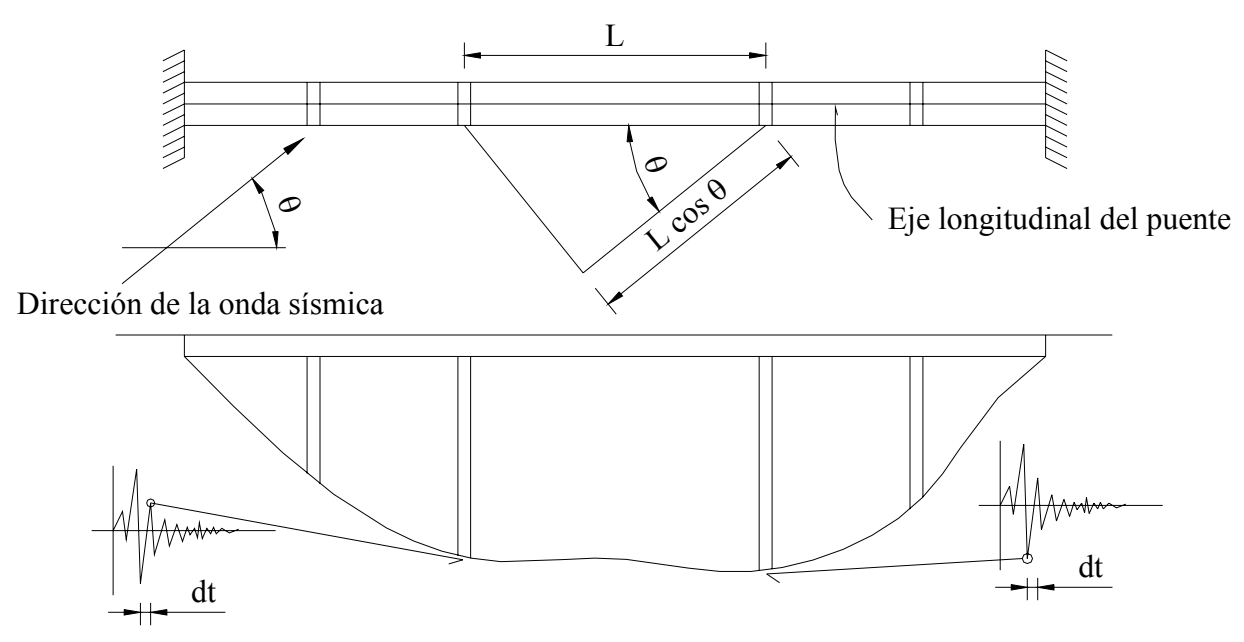

Figura 3. Ilustración del efecto del paso de las ondas (Priestley y otros, 1996).

\section{Efectos de sitio}

Las condiciones locales del suelo en un sitio determinarán las amplitudes y contenido de frecuencias del movimiento sísmico, lo cual es un factor que puede influir de manera importante en la respuesta de una estructura como es el caso de la ciudad de México. A este respecto, la respuesta elástica de una estructura dependerá entre otros factores de la relación entre el periodo del suelo del sitio y el periodo de la estructura. En cuanto a la diferencia en el tiempo de llegada de ondas entre pilas, la velocidad de propagación de las ondas en el suelo en cada sitio modifican el campo incidente, incrementando el tiempo de llegada a la segunda pila.

Debido a la gran separación que existe entre pilas de puentes, se pueden tener suelos con características diferentes debajo de cada una de ellas; en este trabajo, dado que se estudian claros de puentes cortos y medianos $(\mathrm{L}<100 \mathrm{~m})$, se considera el mismo tipo de suelo en todos los apoyos para solamente observar el comportamiento de la misma estructura en sitios diferentes, provocando un desfase en el arribo de las ondas como se explicará más adelante. De acuerdo a lo observado en el valle de México esta consideración será válida prácticamente para cualquier sitio ya que en esos $100 \mathrm{~m}$ de separación entre pilas será muy difícil encontrar variaciones importantes en el perfil del suelo. 


\section{DESCRIPCIÓN DEL ESTUDIO REALIZADO}

En este trabajo se obtienen las diferencias en desplazamientos horizontales en el sentido longitudinal del puente de dos pilas comunes a un mismo claro. Se analizan varios casos de pilas con diferente altura y con diferente separación entre ellas a fin de estudiar el efecto que tienen estos factores en su respuesta; también se considera el efecto que produce el paso de las ondas sísmicas con un desfase en el tiempo $\Delta t$ entre pilas adyacentes, obtenido con la ecuación 1 . De acuerdo a lo anterior, las pilas estarán sometidas a la misma excitación pero afectadas por el desfase en el tiempo de arribo de las ondas. Se asume, por el tamaño de los claros que aquí se estudian $(\mathrm{L}<100 \mathrm{~m})$, que el suelo donde se desplantan las pilas es el mismo por lo que no se considera la influencia de la incoherencia generada por el trayecto de las ondas a través de la estratigrafía del suelo entre las pilas.

Estrada (2004) calculó la respuesta de este tipo de puentes ante varios sismos que se han registrado en México durante los últimos años. Sin embargo, en este estudio se hacen análisis lineales paso a paso usando registros de acelerogramas de varios sitios correspondientes únicamente al terremoto de Michoacán de 1985 por ser el único donde se aprecian respuestas importantes.

\section{Modelado y análisis de las pilas}

Las pilas del puente se idealizan como sistemas de un grado de libertad. En la figura 4, se muestra el tipo de pila estudiada donde se puede observar que el tipo de estructuración usada corresponde a una superestructura típica a base de trabes prefabricadas de concreto presforzado simplemente apoyadas sobre la subestructura, compuesta por pilas aisladas de concreto reforzado. No se tomó en cuenta el posible efecto de cabeceo.

Con la variación del periodo de las pilas obtenemos curvas de comportamiento de los desplazamientos relativos. Este periodo está en función de la longitud del claro entre las pilas y su altura respectiva.

Para los análisis a realizar se usan registros de estaciones correspondientes a sitios con distintas propiedades dinámicas del suelo, ubicados en la costa de Guerrero y en la ciudad de México. Tales sitios se escogieron por presentar grandes demandas de estructuras con periodos de vibrar correspondientes a un rango de interés para los puentes típicos estudiados en este trabajo $(\mathrm{T}<1.3 \mathrm{~s})$.

Los registros corresponden al sismo ocurrido el 19 de septiembre de 1985 ya que durante ese evento se presentó un gran número de daños producto de las acciones a las que fueron sometidas las estructuras. Aunque las estructuras de puentes no sufrieron daño, cabe destacar que la estructuración actual de éstos ha cambiado con respecto a la entonces usada contándose ahora con estructuras de mayor esbeltez donde en muchas ocasiones la superestructura está compuesta por elementos prefabricados simplemente apoyados que tienden a perder su longitud de apoyo. 

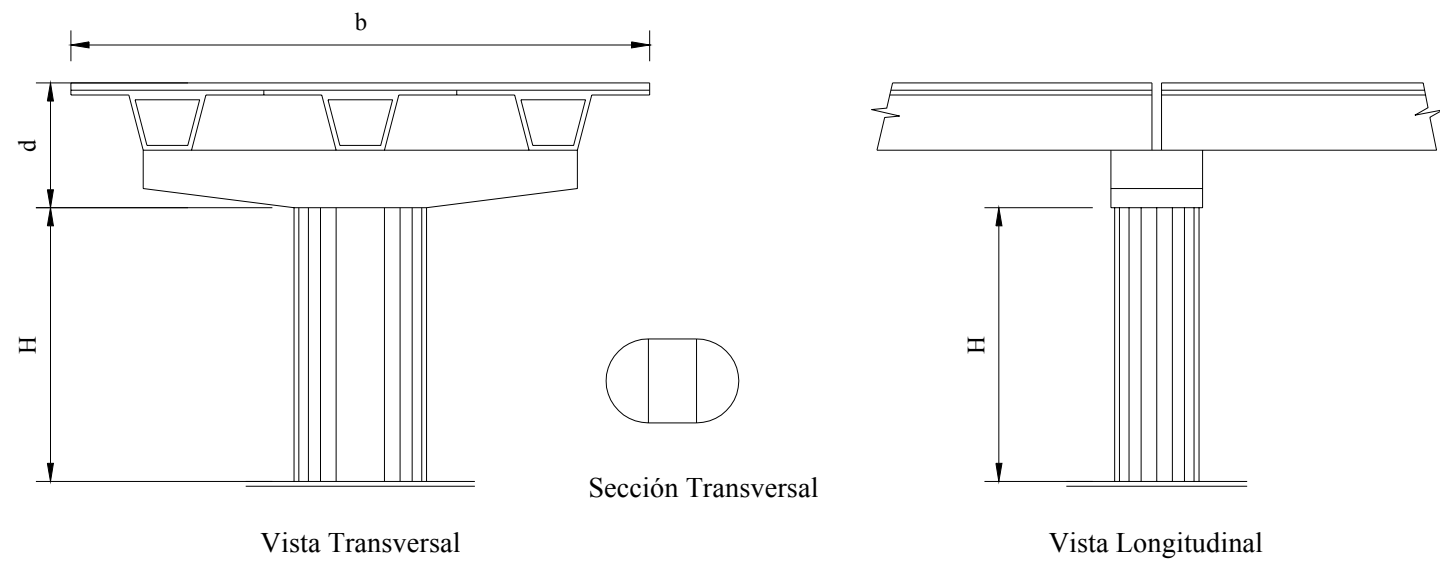

Figura 4. Estructura tipo modelada como un oscilador de un grado de libertad para la obtención de desplazamientos relativos entre pilas.

Los sitios a los cuales corresponden los registros son: La Unión, en la costa de Guerrero, y Central de abastos, SCT y Viveros, en la ciudad de México. En la tabla 1 se presentan los datos del suelo para el análisis en cada sitio (DDF, 1988).

Las longitudes entre pilas a analizar se fijaron de acuerdo a las separaciones entre apoyos típicas de este tipo de puentes y están entre 30 y $80 \mathrm{~m}$.

Tabla 1. Características del suelo de los sitios de estudio

\begin{tabular}{|c|c|c|}
\hline Sitio & Tipo de suelo & Velocidad de la onda sísmica (m/s) \\
\hline La Unión & Firme & 1000 \\
\hline Viveros & Transición & 600 \\
\hline SCT & Blando & 100 \\
\hline Central de Abastos & Blando & 100 \\
\hline
\end{tabular}

\section{Efecto de golpeteo entre trabes}

Al comparar la respuesta de dos pilas independientes el análisis se debe hacer paso a paso y de manera simultánea, puesto que los elementos del puente pueden chocar entre si afectando la respuesta de las pilas.

Considerando que la carga impulsiva producida por el choque es constante como se muestra en la figura 5, la respuesta de un oscilador ante una carga de este tipo, partiendo de un estado de reposo, está dada por las expresiones siguientes, 
Po

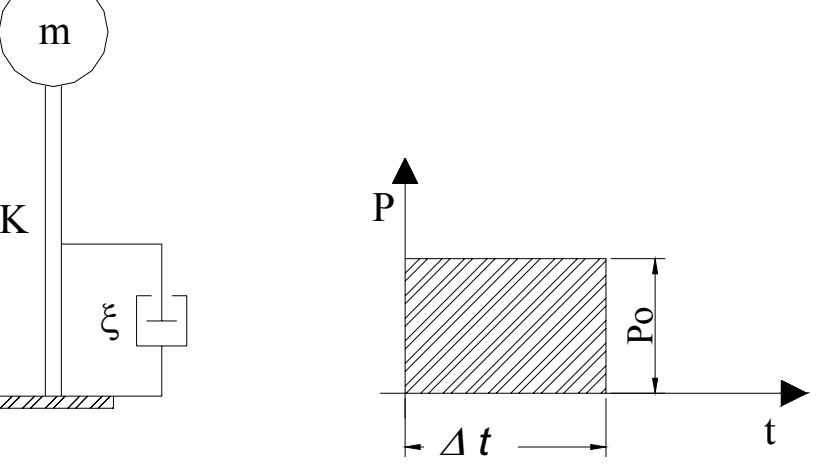

Figura 5. Oscilador sometido a una carga impulsiva.

$$
\begin{aligned}
& X_{p}=\frac{P_{o}}{K} \cdot\left[e^{-\xi \cdot \omega \cdot \Delta t} \cdot\left(-\frac{\xi \cdot \omega}{w_{D}} \cdot \operatorname{sen}\left(w_{D} \cdot \Delta t\right)-\cos \left(w_{D} \cdot \Delta t\right)\right)+1\right] \\
& V_{p}=\frac{P_{o}}{K} \cdot e^{-\xi \cdot \omega \cdot \Delta t} \cdot \frac{\omega^{2}}{w_{D}} \cdot \operatorname{sen}\left(w_{D} \cdot \Delta t\right)
\end{aligned}
$$

donde, $X_{p}$ y $V_{p}$ son el desplazamiento y velocidad de la pila, respectivamente, $w_{D}=\omega \cdot \sqrt{1-\xi^{2}}$ es la frecuencia amortiguada, $\omega, \xi$ y $K$ son la frecuencia, amortiguamiento y rigidez del oscilador, respectivamente, $P_{o}$ es la carga impulsiva y $\Delta t$ es el tiempo de su duración.

Sustituyendo la variable $P_{o}$ en función del impulso constante $\left(P_{o} \Delta t=K_{c}\right)$ en las ecuaciones 2 y 3 , asumiendo que la duración $\Delta t$ de la carga impulsiva $P_{o}$ tiende a cero y aplicando ese límite $(\Delta t \rightarrow$ 0 ) a las ecuaciones 2 y 3 , la respuesta se simplifica y se tiene:

$$
X_{p}=0
$$

$$
V_{p}=\frac{K_{c}}{m}=\frac{P_{o} \cdot \Delta t}{m}
$$

donde $m$ es la masa del oscilador. Lo anterior nos indica que al presentarse el choque, solamente sufrirá modificación la velocidad del oscilador, con respecto a los valores que se tienen antes del mismo, es decir, solo se le da un impulso. Parte del efecto de la fuerza de impacto se verá reflejado en el daño que se produzca en las trabes o elementos de la superestructura.

Existe un procedimiento aproximado para modelar el impacto usado por DesRoches y otros (2002), asumiendo que éste es instantáneo y haciendo uso de la ley de conservación de la 
cantidad de movimiento (momento), así como del coeficiente de restitución ya que el choque no es totalmente elástico. De acuerdo a lo anterior, las velocidades de las masas en cuestión se modifican en el instante del impacto de la siguiente forma:

$$
\begin{aligned}
& v_{1}^{\prime}=v_{1}-(1+e) \cdot \frac{m_{2}\left(v_{1}-v_{2}\right)}{m_{1}+m_{2}} \\
& v_{2}^{\prime}=v_{2}+(1+e) \cdot \frac{m_{1}\left(v_{1}-v_{2}\right)}{m_{1}+m_{2}}
\end{aligned}
$$

donde, $v_{1}{ }_{1}$ y $v_{2}$ son las velocidades de los osciladores 1 y 2 después del impacto, $v_{1}$ y $v_{2}$ son las velocidades de los mismos antes del impacto y $e$ es el coeficiente de restitución, que es la relación entre los impulsos después y antes del impacto y toma valores entre cero y uno. Dicho coeficiente depende del tipo de material de los cuerpos que se impactan y de las condiciones geométricas en que se encuentran tales cuerpos.

\section{Modelado del efecto de golpeteo}

A continuación se presentan las consideraciones que se asumen para obtener los desplazamientos relativos entre las pilas producto del efecto de golpeteo citado anteriormente, con el propósito de simplificar su implementación:

1) No se toma en cuenta la restricción ofrecida por las trabes contiguas a ambos lados del tramo del puente analizado (trabes 1 y 4 mostradas en la figura 6) tal como lo hace Ruangrassamee y otros (2001), por lo que cada elemento de la superestructura del tramo en estudio (trabes 2 y 3 ) solo se considera impactado en el extremo apoyado en la pila 2. Parece razonable asumir que solamente uno de esos extremos tiene libertad de deslizamiento horizontal puesto que es una práctica común analizar, diseñar y construir así este tipo de puentes, como se muestra en la figura 6. El despreciar la restricción de las trabes contiguas obedece a una simplificación razonable del análisis. Es posible que esto sea ligeramente conservador ya que se presentara golpeteo generalizado en todos los componentes del puente, lo que es muy difícil de analizar, redundará en daños locales por golpeteo y menores demandas para la subestructura.

2) Al presentarse el choque entre las masas de la superestructura, las velocidades de éstas serán modificadas de manera aproximada y esto se tomará en cuenta de acuerdo a lo establecido en las ecuaciones 6 y 7. Al coeficiente de restitución se le asigna el valor de 0.8 de acuerdo a DesRoches y otros (2002).

3) Al producirse el choque ente las masas, los desplazamientos de ambas dependerán del sentido de sus desplazamientos antes del choque. Cuando el choque se produzca por el alcance entre los osciladores, es decir, que se desplacen en el mismo sentido, para el cuerpo que es golpeado el desplazamiento en ese instante será el calculado para esa pila sin considerar el choque, mientras que para el otro cuerpo el desplazamiento estará limitado por el del primero más la holgura entre los elementos de la superestructura. Cuando el choque se produce al 
desplazarse ambos osciladores en sentidos opuestos, los desplazamientos estarán restringidos a la holgura entre las trabes. Para este caso, de manera aproximada cada desplazamiento corresponde a una fracción de la holgura entre las trabes y depende de la relación entre el desplazamiento de ambos osciladores calculado sin considerar el choque; la suma de tales desplazamientos en valor absoluto será la holgura entre trabes.

4) Para que lo anterior se cumpla hemos asumido que los valores de las masas de los osciladores deben ser relativamente parecidos entre sí.

5) Se considera una holgura de $10 \mathrm{~cm}$ que es la que se observa típicamente en este tipo de puentes.

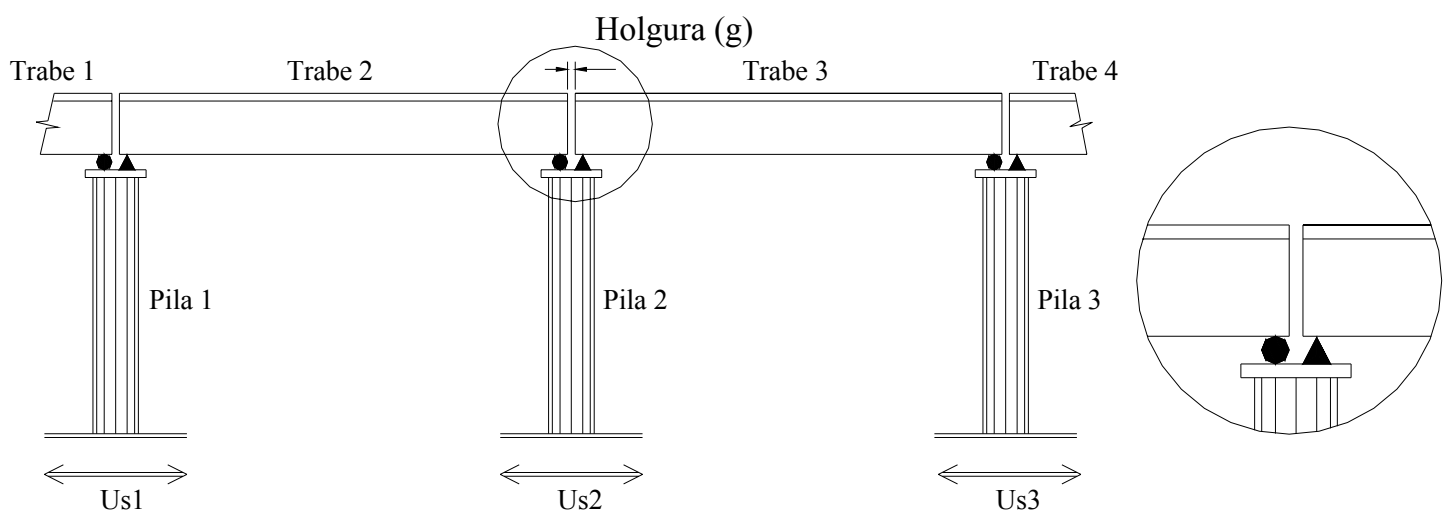

Figura 6. Modelo idealizado del puente.

\section{ANÁLISIS DE LA RESPUESTA OBTENIDA PARA LOS MODELOS DE ESTUDIO}

La variación de los desplazamientos relativos entre las pilas en un sitio es dependiente de la relación del periodo del suelo y de las pilas, así como de la relación de los periodos de las pilas $T_{1}$ y $T_{2}$. Para el caso de sitios en suelo blando estos periodos deben incluir la influencia de la interacción suelo-estructura.

Con el propósito de establecer una relación entre los desplazamientos relativos con respecto al periodo de vibrar de las pilas y a la longitud de separación entre las mismas, se presenta un análisis de tales desplazamientos obtenidos para cada uno de los sitios estudiados para osciladores de un grado de libertad correspondientes a las características de las pilas del puente. Para obtener una distribución de la respuesta que nos permita ajustar los resultados a una expresión en función de las variables mencionadas, se realizan análisis modificando el periodo de la pila más flexible manteniendo constante la longitud de separación entre las pilas y viceversa, así como para varias relaciones de periodos de vibrar de las pilas. En los análisis realizados se considera un ángulo de incidencia de las ondas sísmicas de cero grados, es decir, en el sentido 
longitudinal del puente que es el que produce los mayores desplazamientos relativos entre las pilas y una holgura entre trabes de $10 \mathrm{~cm}$.

\section{Sitio La Unión, Gro.}

La distribución de los desplazamientos relativos de la figura 7 corresponde a la variación de la respuesta obtenida con respecto al periodo de la pila más flexible $\left(T_{1}\right)$. Se muestran resultados para dos longitudes de separación de las pilas $(L=50$ y $80 \mathrm{~m})$ y diferentes relaciones de periodos de las mismas. Los desplazamientos relativos para cuando ambas pilas tienen el mismo periodo $\left(T_{2} / T_{1}=1.0\right)$ no presentan gran variación al modificarse el periodo de vibrar $T_{1}$. En periodos bajos se tienen respuestas iguales a las de periodos mayores a estos debido a que el sitio La Unión está en suelo firme y su respuesta no se modifica para periodos altos. Con respecto a la separación de las pilas, $L$, ambas figuras son muy similares por lo que a partir de estos resultados se puede concluir que esta variable no afecta la respuesta del puente. Esto es debido a que el tiempo producido por el efecto del paso de las ondas sísmicas $\Delta t$ es corto en este tipo de suelo porque las velocidades de las ondas en terreno firme o en roca son muy altas.
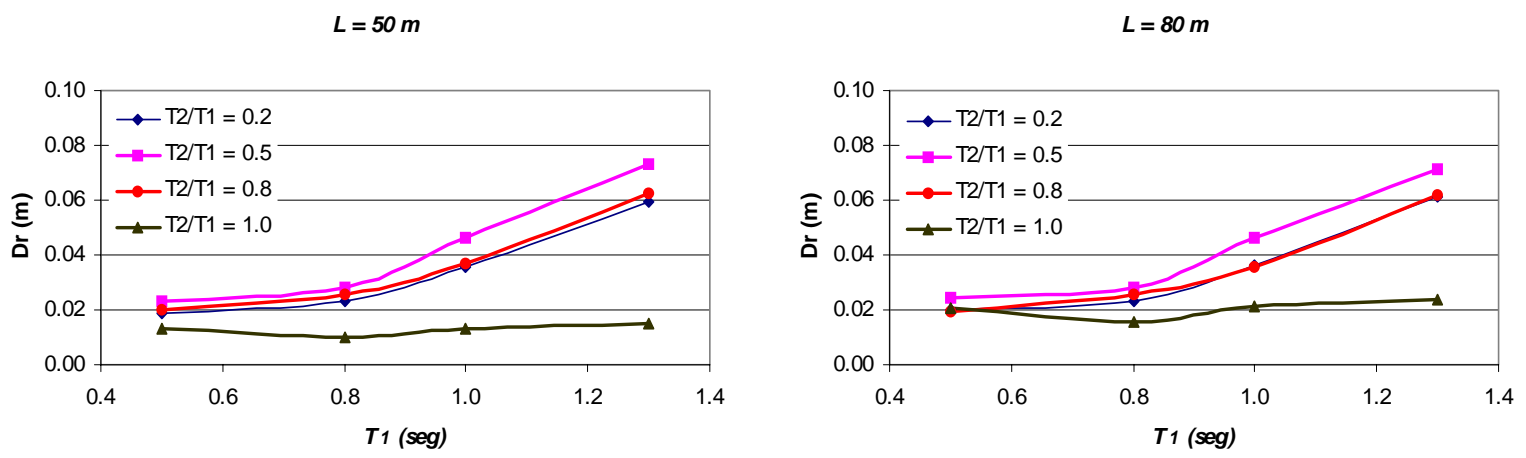

Figura 7. Variación de desplazamientos relativos con respecto al periodo de vibrar de la pila más flexible $\left(T_{1}\right)$ en el sitio La Unión.

Para representar la variación de los desplazamientos relativos en este sitio, se ajustó mediante mínimos cuadrados una curva a la respuesta presentada con respecto al periodo de vibrar de la pila más flexible $T_{1}$, considerando de manera implícita las amplificaciones producidas por el efecto de la diferencia entre los periodos de las pilas. La variación de los desplazamientos relativos en este sitio con respecto al periodo de la pila más flexible se muestra la figura 7; la curva que mejor se ajusta a esta distribución está representada por una ecuación de segundo grado la cual cubre los desplazamientos relativos para cualquier relación de periodos de las pilas, es decir, tomando en cuenta implícitamente las mayores amplificaciones debido a la diferencia en los periodos de las pilas. Los desplazamientos relativos presentan una variación lineal con respecto a la longitud de separación de las pilas, $L$. En resumen, se tiene que,

$$
\operatorname{Dr}_{\text {Unión }}\left(T_{1}, L\right)=0.0002 \cdot L+0.074 \cdot T_{1}^{2}-0.068 \cdot T_{1}+0.037
$$


donde $D r_{\text {Unión }}$ es el máximo desplazamiento relativo entre dos pilas, en metros.

El factor que determina la influencia de la longitud de separación de las pilas en los desplazamientos relativos, no es tan relevante como el periodo de vibrar considerado $\left(T_{1}\right)$ como lo muestra la ecuación 8. Dicha ecuación representa una superficie que muestra una curvatura que define el comportamiento de los desplazamientos relativos con respecto al periodo de vibrar $T_{1}$ correspondiente a la variación de la respuesta calculada analíticamente; tales desplazamientos varían linealmente con respecto a la longitud de separación de las pilas con una pendiente relativamente baja, puesto que el efecto del paso de las ondas es mínimo en este tipo de suelo por presentar una velocidad de las ondas alta, lo anterior se muestra en la figura 8. Esta figura nos indica que la respuesta en periodos bajos es relativamente constante hasta periodos cercanos a 0.80 segundos, debido a que en este tipo de suelo el periodo dominante tiende a cero.

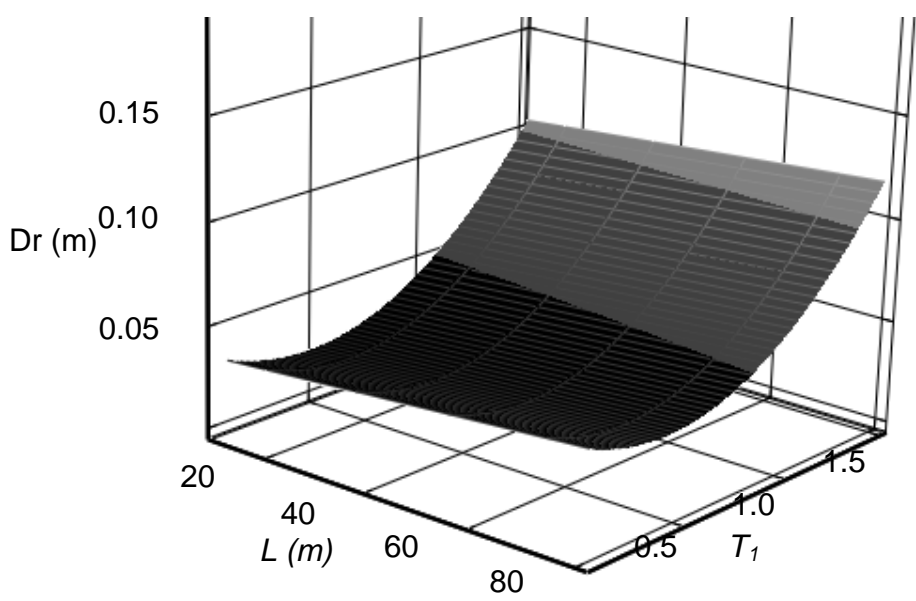

Figura 8. Distribución de desplazamientos relativos en el sitio La Unión.

\section{Sitio Viveros}

Al igual que en la figura 7, la figura 9 muestra la distribución de desplazamientos relativos entre pilas con respecto al periodo $T_{1}$; se muestran resultados para varias longitudes de separación de las pilas y diferentes relaciones de periodos de las mismas. Los desplazamientos relativos para $T_{2} / T_{1}=1.0$ no presentan un cambio significativo con respecto al periodo de vibrar $T_{1}$. En cuanto a la separación de las pilas $L$ para este mismo caso $\left(T_{2} / T_{1}=1.0\right)$, se presenta una variación relativamente lineal con una pendiente baja pero mayor a la presentada en el sitio La Unión debido a que la velocidad de las ondas en este sito es menor que la de aquel. Para la variación de los desplazamientos relativos con respecto a la relación de periodos de vibrar se presenta una amplificación de los mismos que depende del periodo de vibrar de la pila más flexible $T_{1}$ y se mantiene relativamente constante con respecto a la separación de las pilas. Dichas 
amplificaciones no presentan una variación significativa con respecto a la relación de periodos de vibrar de las pilas.
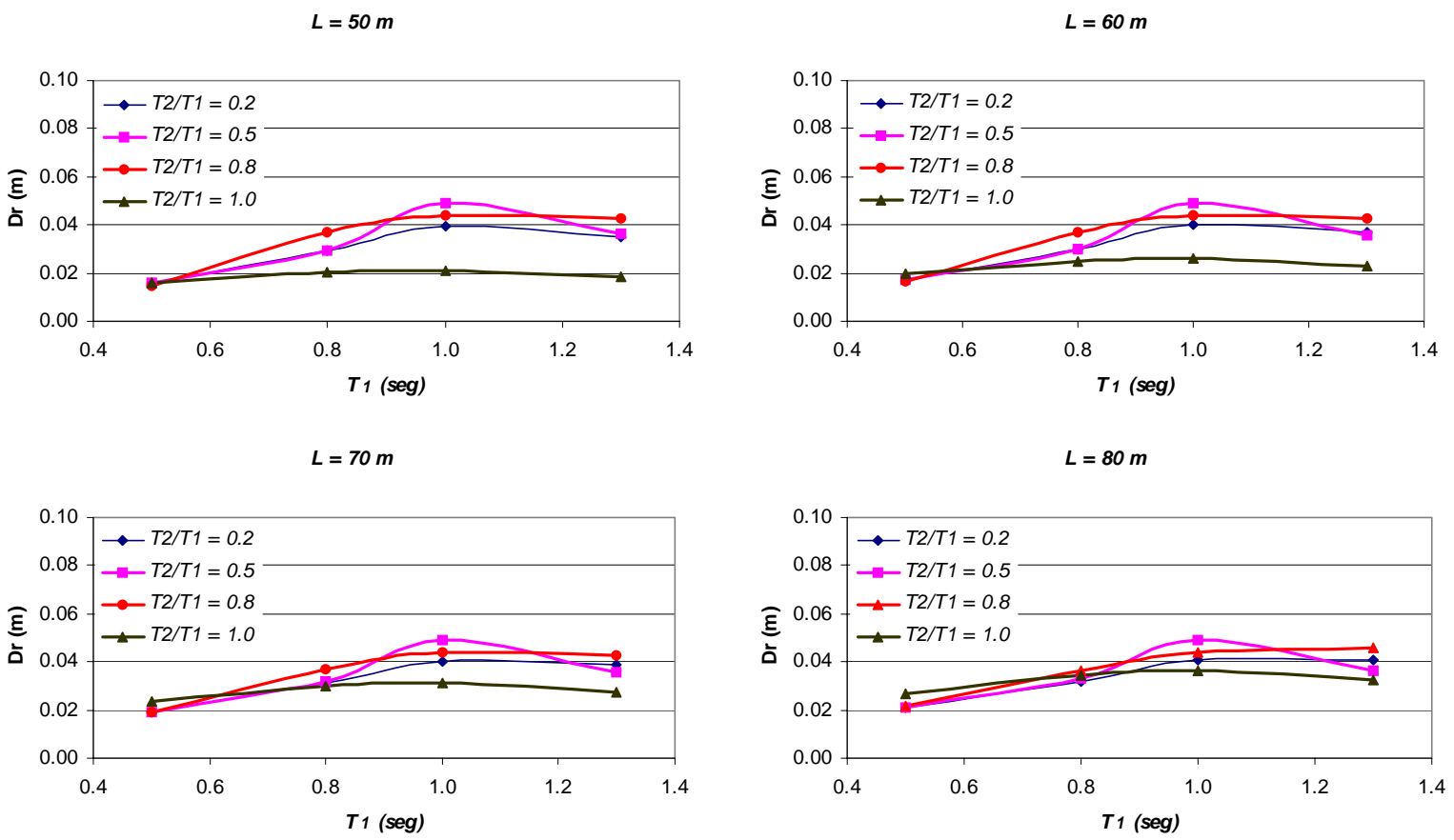

Figura 9. Variación de desplazamientos relativos con respecto al periodo de vibrar de la pila más flexible $\left(T_{1}\right)$ en el sitio Viveros.

Al igual que en el sitio La Unión la variación de la respuesta presentada se asemeja a la curva definida por una ecuación de segundo grado. Realizando el ajuste a los resultados obtenidos de los análisis mostrados en la figura 9 para $L=50 \mathrm{~m}$, se tiene la siguiente ecuación que incluye la contribución de la longitud $L$ :

$$
\operatorname{Dr}_{\text {Viveros }}\left(T_{1}, L\right)=0.0003 \cdot L-0.088 \cdot T_{1}^{2}+0.196 \cdot T_{1}-0.063
$$

La ecuación 9 representa una superficie (figura 10) que muestra una curvatura que define el comportamiento de los desplazamientos relativos con respecto al periodo de vibrar $T_{1}$. La respuesta mayor se presenta en periodos de las pilas cercanos al periodo donde se presentan las mayores aceleraciones espectrales; la variación de tales desplazamientos con respecto a la longitud de separación de las pilas es lineal con una pendiente relativamente baja.

\section{Sitio Secretaría de Comunicaciones y Transportes}

Los resultados obtenidos en este sitio se muestran en la figura 11 y corresponden a la variación de los desplazamientos relativos entre pilas dependientes del periodo de vibrar de la pila más flexible $\left(T_{1}\right)$. Se observa que conforme aumenta la separación de las pilas tiende a perderse la 
dependencia lineal de la respuesta con respecto al periodo de vibrar. Esto es porque el segundo modo en ese sitio está en 0.7 segundos lo que incrementa la respuesta de las pilas en ese valor. Con respecto a la relación de los periodos de las pilas, los desplazamientos relativos máximos se presentan en general cuado los periodos son iguales salvo en el caso de periodos relativamente $\operatorname{largos}(1.3 \mathrm{~s})$ donde se presentan amplificaciones para periodos diferentes $\left(T_{2} / T_{1} \neq 1.0\right)$.

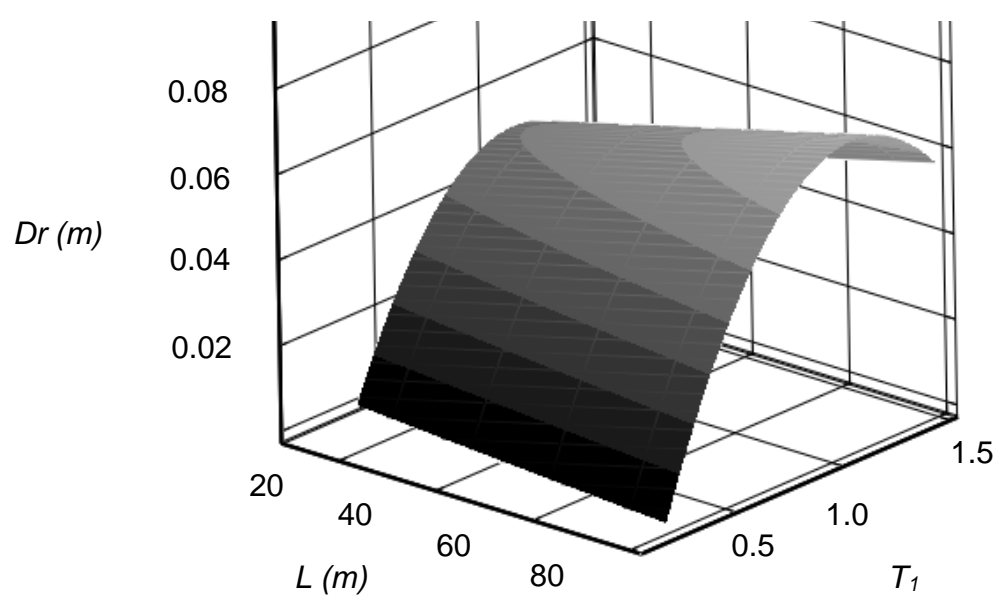

Figura 10. Distribución de desplazamientos relativos para el sitio Viveros.
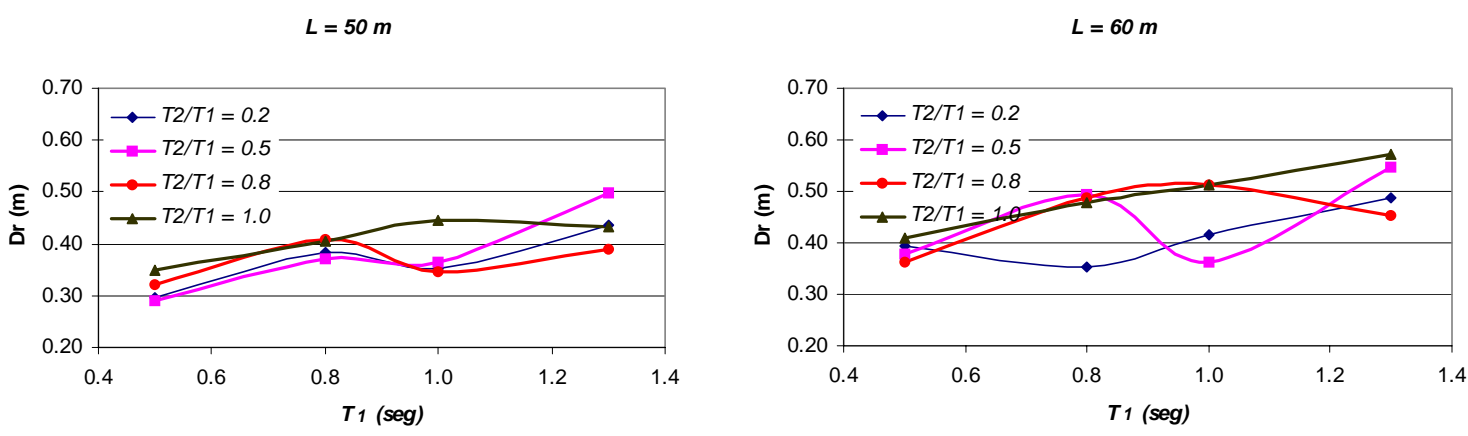

$L=70 \mathrm{~m}$

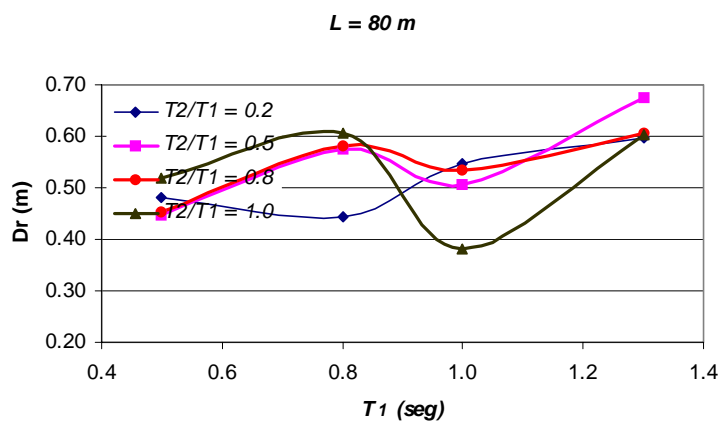

Figura 11. Variación de desplazamientos relativos con respecto al periodo de vibrar de la pila más flexible $\left(T_{1}\right)$ en el sitio SCT. 
En la figura 12 se muestra la distribución de los desplazamientos relativos dependientes de la longitud de separación de las pilas para diferente periodo $T_{1}$, así como para varias relaciones de periodo de las pilas. En todos los casos mostrados se observa una dependencia prácticamente lineal de la respuesta con respecto a la separación de las pilas; para la relación de periodos se presenta la misma tendencia de la figura 11.
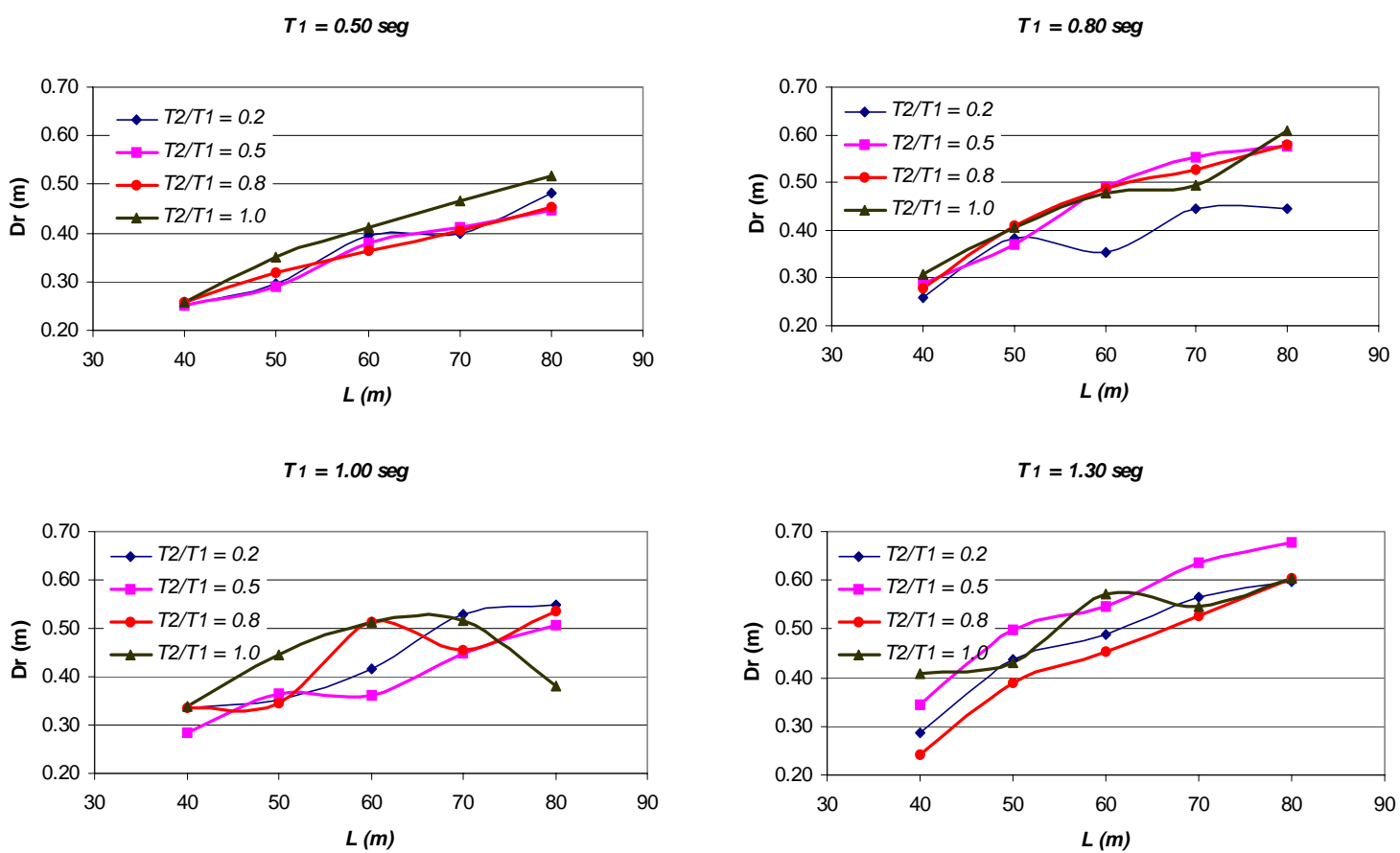

Figura 12. Variación de desplazamientos relativos con respecto a la longitud de separación de las pilas en el sitio SCT.

A partir de la información mostrada en las figuras 11 y 12 se realizó un análisis de regresión lineal múltiple y se calculó una ecuación a partir de $T_{1}$ y $L$ (periodo de vibrar de la pila más flexible y longitud de separación de las pilas) válida para el sitio SCT,

$\operatorname{Dr}_{S C T}\left(T_{1}, L\right)=0.143 \cdot T_{1}+0.006 \cdot L+0.044$

El periodo de vibrar de las pilas y la longitud de separación tienen relativamente el mismo peso en la respuesta. La ecuación 10 no depende de la relación $T_{2} / T_{1}$ porque la dispersión correspondiente es muy grande (Estrada, 2004) y optamos por eliminar ese término.

\section{Sitio Central de Abastos}

Al igual que en SCT para este sitio se obtuvo una distribución de desplazamientos relativos que varían en función del periodo de la pila más flexible y de la longitud de separación de las pilas, similares a las figuras 11 y 12, respectivamente (Estrada, 2004). Para la obtención de una 
expresión que represente los desplazamientos relativos que se pueden presentar en este sitio, se usará la metodología empleada para la obtención de tal expresión para el sitio SCT, por presentar distribuciones de resultados similares, es decir partiendo de la respuesta obtenida para pilas con el mismo periodo de vibrar para luego afectar dicha respuesta con un factor dependiente de la relación de los periodos de vibrar de las pilas.

La ecuación propuesta para Central de Abastos es,

$\operatorname{Dr}_{C D}\left(T_{1}, L\right)=0.127 \cdot T_{1}+0.0037 \cdot L-0.059$

En la ecuación 11, al igual que en la ecuación 10, el cambio en el periodo de vibrar de las pilas tiene relativamente la misma importancia en la respuesta que el cambio de la longitud de separación de las mismas, y el efecto de la diferencia entre los periodos de las pilas tampoco se considera por la poca influencia que tiene en la respuesta.

\section{Comparación de las respuestas obtenidas en los sitios estudiados}

La respuesta de una misma estructura localizada en varios sitios ante un evento sísmico, dependerá de la distancia al epicentro del evento y de las condiciones locales del suelo del sitio. En la figura 13 se presentan los desplazamientos relativos calculados analíticamente mediante un análisis dinámico entre dos pilas de un puente localizado en los sitios aquí estudiados. Comparando tales respuestas observamos la importancia de las condiciones locales del suelo puesto que se trata de la misma estructura analizada. Se muestra que los resultados son mayores en sitios de suelo blando con respecto a los de transición y firme, debido a la magnitud de los desplazamientos del suelo y al efecto del paso de las ondas entre las pilas.
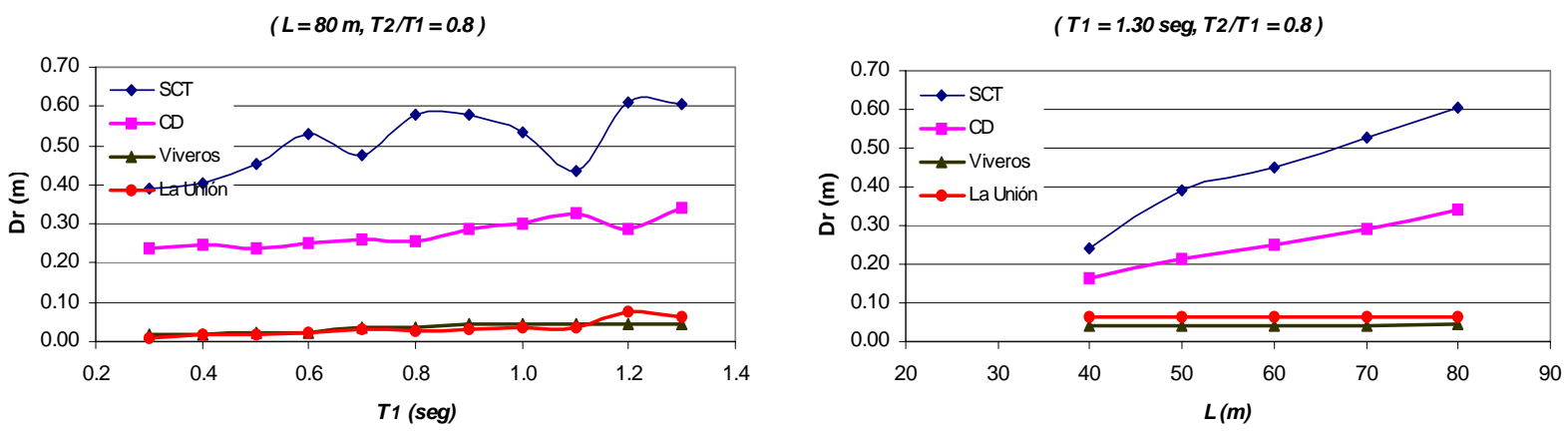

Figura 13. Desplazamientos relativos entre pilas obtenidos dinámicamente en los sitios estudiados.

El comportamiento presentado en los distintos sitios, con respecto a la variación del periodo de vibrar de la pila más flexible $T_{1}$, indica que los máximos desplazamientos relativos dependen en cierta forma de la relación de tal periodo con el periodo del suelo, no obstante al incrementar la flexibilidad de las pilas aumentan tales desplazamientos relativos. En cuanto a la 
separación de las pilas en general se presenta un comportamiento lineal, con una pendiente mayor en suelo blando con respecto a suelo de transición y firme.

\section{Longitud de apoyo en puentes simplemente apoyados}

La longitud de apoyo de los elementos de la superestructura de un puente estructurado a base de claros simplemente apoyados debe garantizar que no se presente la falla del puente por la pérdida de apoyo de la superestructura. Esta longitud depende de varios factores tales como efectos de contracción y expansión térmica, constructivos, es decir se debe tener una longitud de apoyo mínimo y además se deben tomar en cuenta los desplazamientos relativos entre pilas ante un evento sísmico.

En los reglamentos de diseño de puentes tales como el AASTHO (1994) y el CALTRANS (2001) se contemplan expresiones para el cálculo de la longitud mínima de apoyo. En las especificaciones del AASTHO se propone el uso de la siguiente expresión,

$$
L_{\text {Apoyo }}=(200+0.0017 \cdot L+0.0067 \cdot H) \cdot\left(1+0.000125 \cdot S^{2}\right)
$$

donde $L, H$ son la longitud del tablero y la altura de la pila, respectivamente, ambas en mm, $S$ es el ángulo de esviaje en grados; la longitud de apoyo $L_{\text {Apoyo }}$ está dada en mm.

En cuanto al reglamento del CALTRANS, se propone una expresión que depende entre otros factores de efectos térmicos (que determinan la holgura entre las trabes) y de los máximos desplazamientos de las pilas del puente, dicha expresión es,

$$
L_{\text {Apoyo }} \geq\left(h g+\Delta_{e q}+0.10\right)
$$

donde, $L_{\text {Apoyo }}$ está dada en $\mathrm{m}, \mathrm{hg}$ es la holgura entre trabes en $\mathrm{m}, \Delta_{e q}$ es el máximo desplazamiento relativo entre las pilas y está dado por la siguiente expresión,

$$
\Delta_{e q}=\sqrt{\left(\Delta_{D}^{1}\right)^{2}+\left(\Delta_{D}^{2}\right)^{2}}
$$

donde, $\Delta_{D}^{i}$ es el máximo desplazamiento de cada pila obtenido del análisis de la misma.

Para calcular la longitud mínima de apoyo de la superestructura a partir de lo realizado en este estudio, se propone usar una expresión similar a la del CALTRANS, donde las expresiones que definen la variación de los máximos desplazamientos relativos entre las pilas en cada sitio se usen para el cálculo de $\Delta_{\text {eq }}$.

En la figura 14 se muestra con trazos continuos y símbolos la longitud de apoyo mínima obtenida a partir de los máximos desplazamientos relativos calculados con las ecuaciones aquí propuestas para cada sitio estudiado más los $10 \mathrm{~cm}$ mínimos especificados en la ecuación 13. Se muestran algunos resultados obtenidos con la expresión 13 sin considerar la holgura con el fin de 
compararlos con los obtenidos en este estudio, y corresponden a periodos de $0.4,0.6,0.8$ y 1.0 para los sitios La Unión, Viveros, CD y SCT, respectivamente. También se presenta la respuesta obtenida con la expresión propuesta por el AASHTO (1994) para el cálculo de la longitud necesaria de apoyo en este tipo de puentes considerando un ángulo de esviaje de $0^{\circ}$.

Los resultados obtenidos con la expresión del AASHTO son mayores a los obtenidos en los sitios de suelo firme (La Unión) y de transición (Viveros); en el caso el sitio CD la longitud de apoyo es muy parecida a la obtenida con la ecuación 12, pero para el sitio SCT tal longitud de apoyo es mucho mayor a la obtenida con dicha ecuación. Con respecto a los casos de comparación presentados con la ecuación del CALTRANS (círculos rellenos), en general se calculan demandas conservadoras pero no en suelo blando donde solo para el sitio CD y para periodos largos dicha expresión resulta adecuada.

$(L=80 \mathrm{~m}, T 2 / T 1=0.8)$

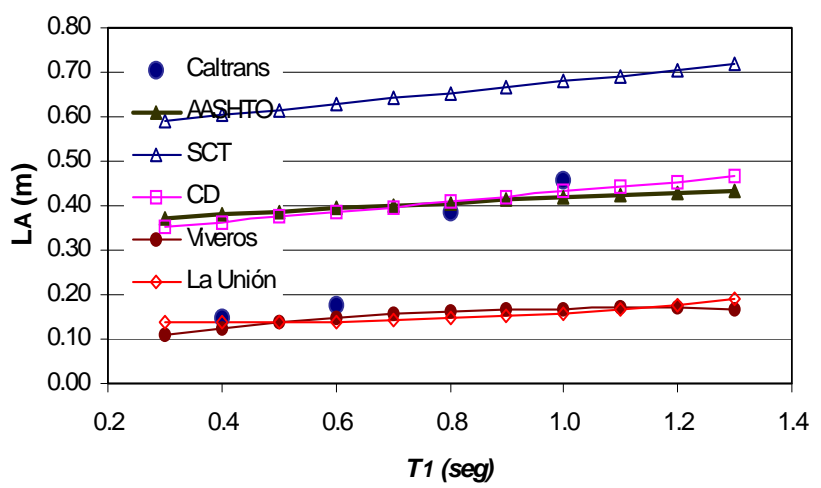

$(T 1=1.30 \mathrm{seg}, T 2 / T 1=0.8)$

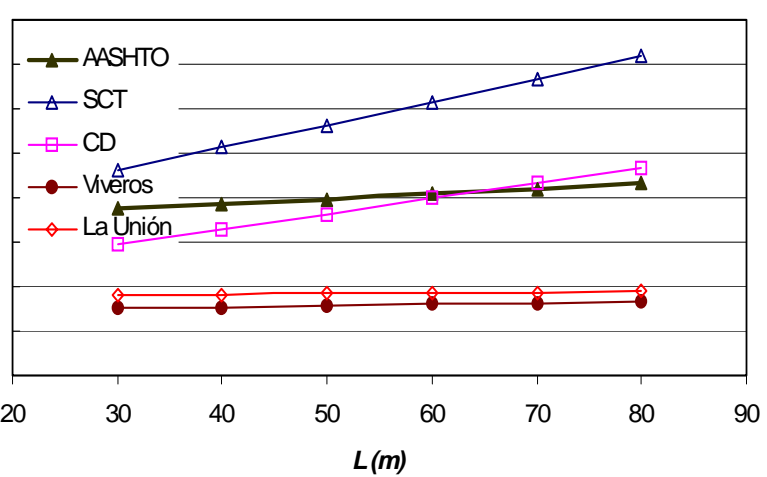

Figura 14. Longitud mínima de apoyo de elementos de la superestructura en puentes simplemente apoyados para cada sitio estudiado.

\section{CONCLUSIONES}

En este trabajo se obtuvieron los máximos desplazamientos relativos que se pueden presentar en pilas adyacentes a un claro en puentes típicamente urbanos en la dirección longitudinal de los mismos localizados en varios sitios ante un evento sísmico. Tales desplazamientos relativos se obtuvieron de un análisis dinámico paso a paso en el cual se analizan simultáneamente dos modelos de pilas de este tipo de puentes considerándolas como osciladores de un grado de libertad. Se toma en cuenta la diferencia en el tiempo de arribo de las ondas en cada pila generada por la separación de éstas como una aproximación a la variación en el movimiento del suelo, así como el efecto de golpeteo entre las trabes de los claros del puente estudiados.

En los sitios correspondientes a suelo de transición y firme, Viveros y La Unión respectivamente, los máximos desplazamientos relativos dependen principalmente del periodo de vibrar de las pilas, de la relación entre estos y del periodo del suelo del sitio (en el caso de transición). Con respecto a la longitud de separación de las pilas, se presenta un comportamiento 
lineal con una pendiente relativamente baja siendo mayor en Viveros, ya que en estos sitios se tiene una diferencia entre el tiempo de arribo a las pilas relativamente corto. En cuanto a la relación de periodos de las pilas, se presenta una amplificación significativa de los desplazamientos relativos cuando existen diferencias entre tales periodos con respecto al caso en que los periodos son iguales.

Para los sitios localizados en suelo blando como SCT y CD los máximos desplazamientos relativos presentados muestran el siguiente comportamiento. Con respecto al periodo de vibrar de la pila más flexible $T_{1}$, se presenta una variación lineal de la respuesta debido a que el periodo del suelo está por encima del intervalo de periodos estudiado. Al variar la longitud de separación de las pilas $L$ se presenta un dependencia lineal, ya que al aumentar la longitud se tiene un mayor intervalo de tiempo $\Delta t$ entre el registro de las ondas sísmicas en las pilas. En cuanto a la relación de los periodos de las pilas no representa un factor relevante en la respuesta como en el caso de suelo firme debido a la mayor importancia del efecto que produce el paso de las ondas.

Las ecuaciones usadas en manuales de diseño tales como AASTHO y CALTRANS para el cálculo de la longitud mínima de apoyo ofrecen resultados muy parecidos a los obtenidos con las expresiones aquí propuestas sobre todo en sitios de suelo firme y transición. Para el caso de suelos blandos en ciertos sitios se presentan diferencias muy marcadas puesto que tales ecuaciones en general son usadas en sitios con suelos con características muy diferentes a las condiciones del suelo blando de la ciudad de México.

En general los resultados obtenidos para los diferentes sitios y bajo las condiciones de modelado y análisis aquí empleadas reflejan que existe la posibilidad de falla por pérdida de apoyo de la superestructura en este tipo de puentes con claros largos, ubicados en zonas de suelo blando y con periodos de pilas relativamente largos en los cuales no se cuente con la longitud necesaria de apoyo de la superestructura, además del consiguiente daño por golpeteo independientemente de tal falla.

El estudio presentado muestra resultados obtenidos para un evento sísmico de subducción, el sismo de Michoacán de 1985, cuya importancia ha sido enorme. Un sismo cuya fuente sea distinta producirá efectos diferentes en los sitios y puentes estudiados. Los sismos de falla normal presentan un mayor contenido de altas frecuencias que produce mayores amplificaciones espectrales en terreno firme y de transición con respecto a las amplificaciones presentadas durante sismos de subducción en esos sitios, pero aún no contamos con registros de intensidad importante para este tipo de fuentes. Por tal motivo, para profundizar en este tipo de estudios es necesario realizar análisis con eventos sísmicos con fuentes sismológicas diferentes aunque sea para acelerogramas simulados para obtener resultados que nos permitan evaluar de una manera más general el comportamiento de este tipo de estructuras. Asimismo, investigaciones futuras deben incluir el estudio de otro tipo de estructuraciones de puentes incluyendo irregularidades tanto en planta como en elevación, así como incluir en un solo modelo todos los componentes del puente y la interrelación que pueda existir entre ellos. 


\section{RECONOCIMIENTOS}

Se hace un especial reconocimiento al Consejo Nacional de Ciencia y Tecnología CONACYT, por el apoyo otorgado al primer autor para la realización de estudios de maestría, así como por la promoción y fomento de la investigación científica. Al Gobierno del Distrito Federal por el patrocinio del proyecto de investigación "Análisis de la respuesta de conjunto con tramos que incluyen varios apoyos considerando movimientos fuera de fase". Agradecemos la participación del Ing. Vicente Orozco en algunas partes de este trabajo.

\section{REFERENCIAS}

AASHTO (1994), "LRFD bridge design specifications", American Association of State Highway and Transportation Officials, Washington, D.C., First Edition, Cap. 4.

CALTRANS (2001), "Seismic design criteria", California Department of Transportation Division of Engineering Services, California, Version 1.2, Cap. 7, pp. 8.

DDF (1988), "Estudio sobre sismicidad en el valle de México", Departamento del Distrito Federal, Secretaría General de Obras, PNUD, HABITAT, Parte 1, Cap. III, 1988.

DesRoches, R y S Muthukumar (2002), "Effect of pounding and restrainers on seismic response of multiple - frame bridges", ASCE Journal of Structural Engineering, Vol. 128, No. 7, pp.860869.

Estrada, R J L (2004), "Desplazamientos relativos en pilas de puentes urbanos", Tesis de Maestría, DEPFI, UNAM, México, septiembre.

Hao, H (1998), "A parametric study of the required seating length for bridge decks during earthquake”, Earthquake Engineering and Structural Dynamics, Vol. 27, pp. 91-103.

Malhotra, P K (1998), "Dynamics of pounding at expansion joints of concrete bridges", ASCE Journal of Engineering Mechanics, Vol. 124, No. 7, julio.

Priestley, M J N, F Seible y G M Calvi (1996), Seismic design and retrofit of bridges, John Wiley \& Sons, New York.

Reinoso, E (2002), Scattering of Seismic Waves with Applications to the Mexico City Valley, WIT Press, Southampton Inglaterra, ISBN 1-85312-833-3, 200pp.

Roullé, A (2004), "El movimiento sísmico en el valle de México: Análisis de datos de un arreglo 3D de pequeña apertura en la colonia Roma", Tesis Doctoral, Posgrado en Ciencias de la Tierra Instituto de Geofísica, UNAM, junio.

Ruangrassamee, A y K Kawashima (2001), "Relative displacement response spectra with pounding effect", Earthquake Engineering and Structural Dynamics, Vol. 30, pp. 1511-1538. 